\title{
Generating Maximal Entanglement between Spectrally Distinct Solid-State Emitters
}

\author{
Hurst, David L.; Joanesarson, Kristoffer B.; lles-Smith, Jake; Mørk, Jesper; Kok, Pieter
}

Published in:

Physical Review Letters

Link to article, DOI:

10.1103/PhysRevLett.123.023603

Publication date:

2019

Document Version

Publisher's PDF, also known as Version of record

Link back to DTU Orbit

Citation (APA):

Hurst, D. L., Joanesarson, K. B., lles-Smith, J., Mørk, J., \& Kok, P. (2019). Generating Maximal Entanglement between Spectrally Distinct Solid-State Emitters. Physical Review Letters, 123(2), [023603].

https://doi.org/10.1103/PhysRevLett.123.023603

\section{General rights}

Copyright and moral rights for the publications made accessible in the public portal are retained by the authors and/or other copyright owners and it is a condition of accessing publications that users recognise and abide by the legal requirements associated with these rights.

- Users may download and print one copy of any publication from the public portal for the purpose of private study or research.

- You may not further distribute the material or use it for any profit-making activity or commercial gain

- You may freely distribute the URL identifying the publication in the public portal 


\title{
Generating Maximal Entanglement between Spectrally Distinct Solid-State Emitters
}

\author{
David L. Hurst, ${ }^{1, *}$ Kristoffer B. Joanesarson, ${ }^{1,2, *}$ Jake Iles-Smith, ${ }^{1}$ Jesper Mørk, ${ }^{2}$ and Pieter Kok ${ }^{1, \dagger}$ \\ ${ }^{1}$ Physics and Astronomy, University of Sheffield, Hounsfield Road, Sheffield, S3 7RH, United Kingdom \\ ${ }^{2}$ Department of Photonics Engineering, Technical University of Denmark, Ørsteds Plads, DK-2800 Kgs. Lyngby, Denmark
}

(Received 12 February 2019; published 9 July 2019)

\begin{abstract}
We show how to create maximal entanglement between spectrally distinct solid-state emitters embedded in a waveguide interferometer. By revealing the rich underlying structure of multiphoton scattering in emitters, we show that a two-photon input state can generate deterministic maximal entanglement even for emitters with significantly different transition energies and linewidths. The optimal frequency of the input is determined by two competing processes: which-path erasure and interaction strength. We find that smaller spectral overlap can be overcome with higher photon numbers, and quasimonochromatic photons are optimal for entanglement generation. Our work provides a new methodology for solid-state entanglement generation, where the requirement for perfectly matched emitters can be relaxed in favor of optical state optimization.
\end{abstract}

DOI: $10.1103 /$ PhysRevLett.123.023603

Quantum technologies promise dramatic improvements in computing and communication by utilizing quantum entanglement between qubits [1]. Although many promising quantum technology architectures have emerged over the past two decades, none are free from the practical challenges presented by high-fidelity quantum control and scalability. For example, superconducting circuit implementations enjoy excellent coherence properties but operate slowly [2], while trapped ion qubits can be prepared with almost unit fidelity but are difficult to scale [3]. Solidstate architectures, such as optically coupled spin systems, compete on speed and scalability. They include semiconductor quantum dots and nitrogen-vacancy centers. Large optical nonlinearities in solid-state systems are now very common [4-6], and solid-state emitters are readily integrated into complex photonic structures, further enhancing the light-matter interaction [7]. However, there are many challenges still to overcome. For example, charge noise and phonon scattering have limited the size of the optical nonlinearities observed thus far [4].

Another major drawback to solid-state emitters is that the central energies and lifetimes of their transitions are highly dependent on the fabrication process, and vary significantly both across and within samples [8]. Known methods for entangling solid-state qubits require emitters with identical energies to facilitate path-erasure techniques $[9,10]$. This adds a practically insurmountable overhead to the process of matching multiple solid-state qubits for creating large entangled states [11]. Stark shifting and strain tuning the emitter transitions has been employed to tune solid-state emitters onto resonance [12-14], but this requires a substantial technical overhead, and arbitrary emitters in a sample cannot in general be tuned onto resonance. Here, we propose a process for generating entanglement that is robust against spectral variations in the emitters' transition energies and linewidths. We show that photons, linear optics, and photon counting suffice to create deterministic entanglement between imperfectly matched emitters, revealing a rich underlying structure of multiphoton scattering off two nonidentical emitters. While many challenges remain, this work removes a major obstacle to a scalable solid-state quantum technology architecture.

Our setup is shown in Fig. 1. Two solid-state emitters each have an $L$-type level structure, with two stable lowlying spin states $(|\uparrow\rangle,|\downarrow\rangle)$, and a dipole transition that

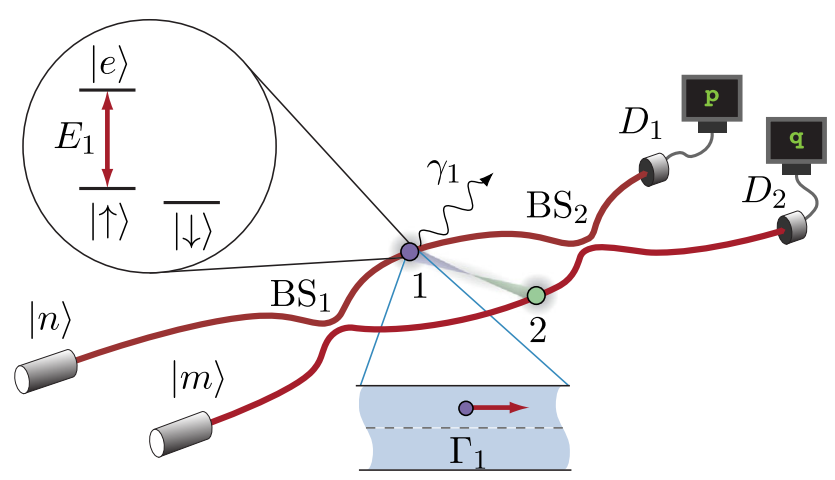

FIG. 1. Waveguide Mach-Zehnder interferometer with emitters embedded at positions 1 and 2, and with $L$-type level structures shown in the inset. The excited state $|e\rangle$ is coupled to a spin qubit state (e.g., $|\uparrow\rangle)$ with transition energy $E_{\alpha}(\alpha=1,2)$, circular polarization, and linewidth $\Gamma_{\alpha}$. The emitters are placed off axis in the waveguide at $c$ points, such that circularly polarized light scatters only in the forward direction. The loss rate from the guided mode is $\gamma_{\alpha}$. Fock states $|n, m\rangle$ are injected into the interferometer, and detectors $D_{1}$ and $D_{2}$ record a photon number detector signature $(p, q)$. 
couples a spin state to an excited state $|e\rangle$. The transition energy for emitter $\alpha=1,2$ is $E_{\alpha}$, and the polarization is circular due to selection rules. The emitters are initially prepared in the product state $(|\uparrow\rangle+|\downarrow\rangle)(|\uparrow\rangle+|\downarrow\rangle) / 2$ and embedded in a waveguide Mach-Zehnder interferometer at $c$ points, where perfect correlation between propagation direction and circular polarization occurs [15]. Consequently, the emitters scatter circularly polarized light only in the forward direction, as was demonstrated recently using semiconductor quantum dots under an applied magnetic field [16-19]. For a lossless waveguide, the emitter will impart a $\pi$-phase shift to each photon that is on resonance with the transition [20-23]. The input to the interferometer is a two-mode Fock state $|n, m\rangle$, and the detectors $D_{1}$ and $D_{2}$ produce classical signatures $(p, q)$ indicating the presence of $p$ and $q$ photons, respectively.

Assuming the emitters are identical, a single monochromatic resonant photon injected into either one of the input arms of the interferometer will scatter from one of the emitters, and after the final beam splitter the light-matter degrees of freedom are in the multipartite entangled state $\left(\left|\Phi^{-}\right\rangle \otimes|1,0\rangle-\left|\Psi^{-}\right\rangle \otimes|0,1\rangle\right) / \sqrt{2}$, where $|0,1\rangle$ and $|1,0\rangle$ is the two-mode single-photon state at the interferometer output. A photon detector signature $(1,0)$ or $(0,1)$ heralds the maximally entangled spin state $\left|\Phi^{-}\right\rangle=$ $(|\uparrow, \uparrow\rangle-|\downarrow, \downarrow\rangle) / \sqrt{2}$ or $\left|\Psi^{-}\right\rangle=(|\uparrow, \downarrow\rangle-|\downarrow, \uparrow\rangle) / \sqrt{2}$. This method can be made robust to photon loss provided that the emitter detuning is only a fraction of the emitter linewidth. Mahmoodian et al. showed how this can form a building block for distributed quantum computing [24].

In practice, both the linewidths and transition energies vary significantly between solid-state emitters, and it was generally assumed that this prohibits the creation of perfect entanglement using linear optics and photodetection. In this case, the input photon can no longer be resonant with both emitters simultaneously. With $\hbar \omega$ the single-photon energy, $\Gamma_{\alpha}$ the unidirectional emission rate of emitter $\alpha=1,2$ into the waveguide, and $\gamma_{\alpha}$ the corresponding coupling to nonguided modes, the scattering process is described by the transmission coefficient [25]:

$$
t_{\alpha}(\omega)=\frac{\hbar \omega-E_{\alpha}-i \hbar\left(\Gamma_{\alpha}-\gamma_{\alpha}\right) / 2}{\hbar \omega-E_{\alpha}+i \hbar\left(\Gamma_{\alpha}+\gamma_{\alpha}\right) / 2} .
$$

We characterize the emitter loss by $\beta_{\alpha} \equiv \Gamma_{\alpha} /\left(\Gamma_{\alpha}+\gamma_{\alpha}\right)$. For nonzero emitter detuning $\delta \equiv E_{2}-E_{1}, t_{\alpha}(\omega)$ ceases to be a $\pi$-phase shift, and for $\beta_{\alpha}<1, t_{\alpha}(\omega)$ is no longer a pure phase shift. The setup then does not create maximally entangled states deterministically anymore. Nevertheless, we will now demonstrate how tailoring the optical input state $|n, m\rangle$ into the Mach-Zehnder interferometer leads to deterministic maximal entanglement between two spectrally distinct emitters.

In general, a detector signature $(p, q)$ indicates that the two emitters are in a mixed entangled state. We use the concurrence $\mathcal{C}(\rho)$ for a two-qubit state $\rho$ to quantify this entanglement [26]. Each signature $(p, q)$ occurs with probability $\operatorname{Pr}(p, q)$ and results in an emitter state $\rho_{(p, q)}$, leading to a concurrence $\mathcal{C}\left(\rho_{(p, q)}\right)$. We define the average concurrence as

$$
\mathcal{C}_{\mathrm{av}} \equiv \sum_{(p, q)} \operatorname{Pr}(p, q) \mathcal{C}\left(\rho_{(p, q)}\right)
$$

This is an appropriate figure of merit, since it provides a lower bound for the amount of entanglement expected from a given experiment without postselection. The entanglement in the two-qubit state can be increased by discarding measurement outcomes corresponding to below-average concurrences. This comes at the expense of the rate of entanglement generation.

The amount of entanglement that can be generated between the two spectrally distinct emitters with a single probe photon is shown in Fig. 2. The single-photon protocol is analyzed using linear optics transformations

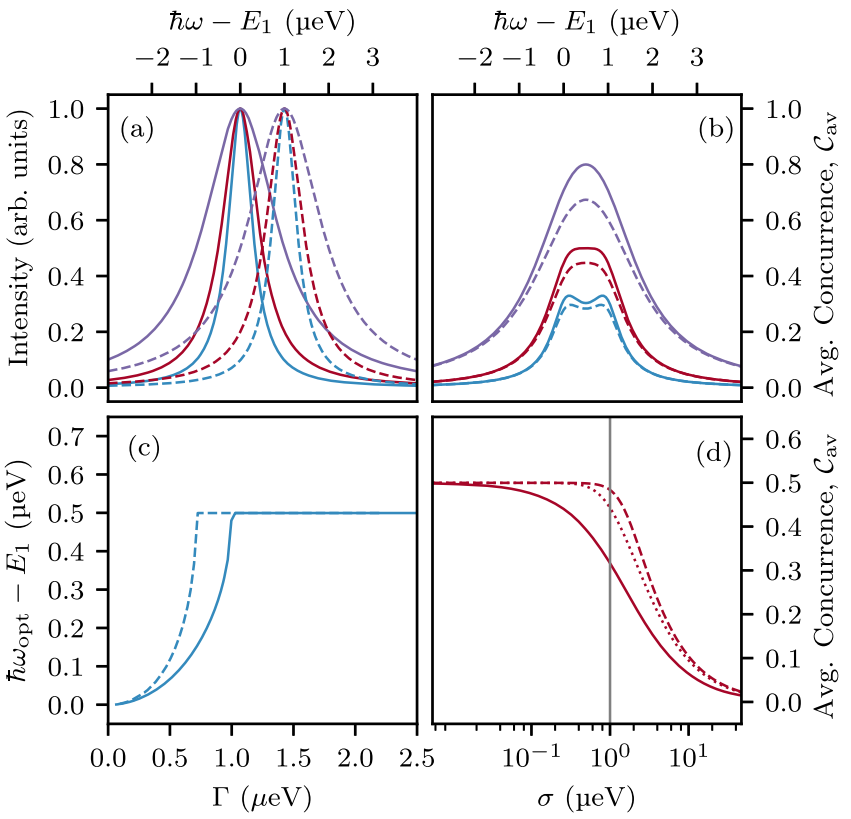

FIG. 2. Single-photon (e.g., $|1,0\rangle$ ) entanglement generation for a pair of detuned $L$-type emitters with equal linewidth $\Gamma$ and energies $E_{1}$ and $E_{2}=E_{1}+\delta$, where $\delta=1.0 \mu \mathrm{eV}$. (a) Lorentzian spectra for emitters with energies $E_{1}$ in arbitrary units (a.u.) (solid lines), $E_{2}$ (dashed lines), and emitter linewidths $0.66 \mu \mathrm{eV}$ (blue lines), $1.0 \mu \mathrm{eV}$ (red lines), and $2.0 \mu \mathrm{eV}$ (purple lines). (b) Average concurrence versus monochromatic single-photon energy without loss $\left(\beta=\beta_{1}=\beta_{2}=1\right.$, solid lines) and with loss $(\beta=0.9$, dashed lines). Line colors as in (a). (c) Location of optimum single-photon energy $\hbar \omega_{\text {opt }}$ for maximum $\mathcal{C}_{\mathrm{av}}$ as a function of emitter linewidth $\Gamma$ for a monochromatic input without loss (solid line) and with $\beta=0.9$ (dashed line). (d) $\mathcal{C}_{\mathrm{av}}$ for Lorentzian (solid line), Gaussian (dotted line), and square (dashed line) singlephoton envelopes as a function of FWHM pulse width $\sigma$. Here, $\hbar \omega=\left(E_{1}+E_{2}\right) / 2$ and $\Gamma=1.0 \mu \mathrm{eV}$. The vertical line indicates the linewidth of the emitters. 
[24], while a multiphoton input requires taking into account the nonlinear nature of the interaction [22] [see Supplemental Material (SM) for details [27] ]. As expected, for spectrally distinct emitters the average concurrence does not reach its maximal value [Fig. 2(b)]. The amount of entanglement is determined by two competing processes. On the one hand, which-path information for the probe photon must be erased, while at the same time the phase shift induced by the photon scattering event must be maximized. Tuning closer to either emitter increases the relative phase shift but also imparts a degree of path information onto the probe, as the light-matter interaction is now stronger for one of the emitters. For emitters with finite detuning and linewidth it is not obvious which photon energy maximizes the average concurrence. Three emitter linewidths are shown in Fig. 2(a), and Fig. 2(b) shows the corresponding $\mathcal{C}_{\mathrm{av}}$. The linewidths shown correspond to emitters with $1,0.66$, and 0.33 ns lifetimes, typical of semiconductor QDs benefiting from modest Purcell enhancements [28]. Increasing the linewidth of the emitters leads to a larger spectral overlap, thereby erasing some of the which-path information and increasing $\mathcal{C}_{\mathrm{av}}$. Figure 2(c) shows the optimal frequency of the input photon that maximizes $\mathcal{C}_{\mathrm{av}}$. For narrow linewidths it is preferable to tune the photon energy away from the mean emitter energy $\left(\hbar \omega-E_{1}=0.5 \mu \mathrm{eV}\right.$ for $\left.\delta=1.0 \mu \mathrm{eV}\right)$, and towards resonance with one of the emitters. Though this reduces the concurrence in the state heralded by a click at detector $D_{2}$, it does increase the probability of a successful scattering event.

One may expect that a photon with a wide frequency bandwidth that overlaps with both emitters will improve the entanglement generation. Figure 2(d) shows the average concurrence for a single probe photon with Lorentzian, Gaussian, and square spectral profiles, centered at $\hbar \omega=E_{1}+\delta / 2$, as a function of the photon bandwidth. We find that increasing the bandwidth of the input photon only degrades the average concurrence, and a narrow band probe is always preferable. We attribute this to the reduced temporal extent of the photon at larger bandwidths, which increases the probability of exciting the emitter, and thus the fraction of light emitted incoherently through spontaneous emission. This reduction is particularly noticeable for a Lorentzian wave packet, where a close spectral match with the emitter increases the excitation probability. We conclude that for given emitter detuning and linewidths, the maximum $\mathcal{C}_{\text {av }}$ of the single-photon case is limited by the competing requirements of maximizing the induced phase shift and path erasure.

Next, we consider whether two photons can increase the average concurrence. Consider an input state of two identical monochromatic photons $|n, m\rangle=|1,1\rangle$ entering the interferometer. They will evolve into a two-photon NOON state $(|2,0\rangle-|0,2\rangle) / \sqrt{2}$ via Hong-Ou-Mandel interference on the first beam splitter $[29,30]$ and interact

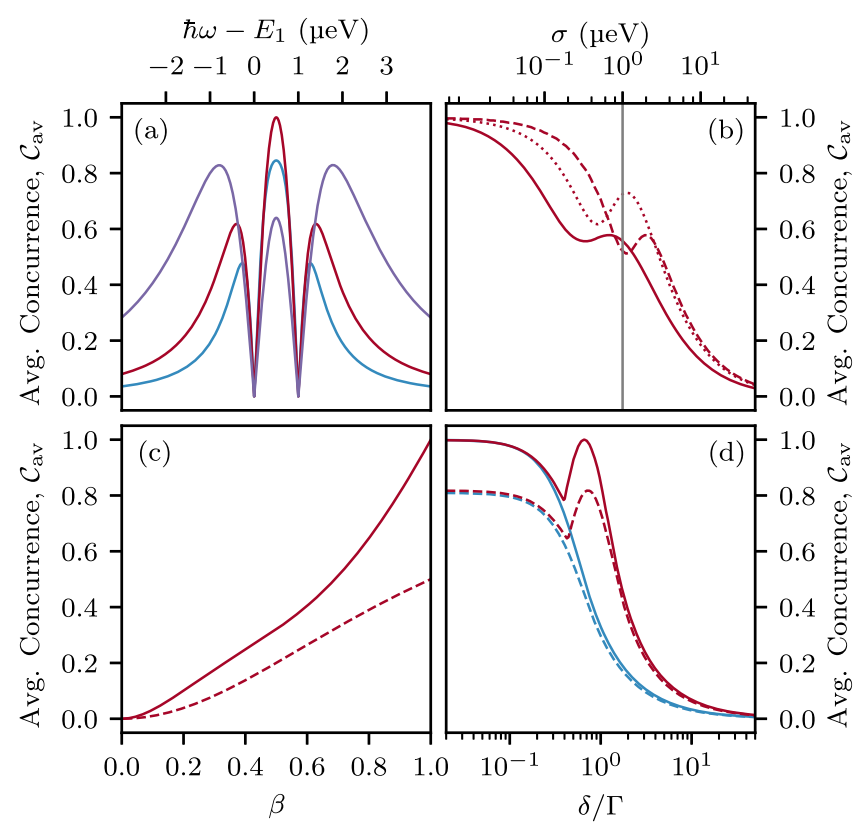

FIG. 3. Two-photon (i.e., $|1,1\rangle$ ) entanglement generation for a pair of detuned $L$-type emitters with equal linewidth $\Gamma$ and energies $E_{1}$ and $E_{2}=E_{1}+\delta$, where $\delta=1.0 \mu \mathrm{eV}$. (a) Average concurrence versus monochromatic two-photon energy. The emitters have equal linewidth of $0.66 \mu \mathrm{eV}$ (blue line), $1.0 \mu \mathrm{eV}$ (red line), and $2.0 \mu \mathrm{eV}$ (purple line). (b) $\mathcal{C}_{\mathrm{av}}$ for Lorentzian (solid line), Gaussian (dotted line), and square (dashed line) singlephoton envelopes as a function of FWHM pulse width $\sigma$. Here, $\hbar \omega=\left(E_{1}+E_{2}\right) / 2$ and $\Gamma=1.0 \mu \mathrm{eV}$. The vertical line indicates the linewidth of the emitters. (c) Average concurrence as a function of $\beta=\beta_{1}=\beta_{2}$ for a monochromatic two-photon pulse (solid line) and monochromatic single-photon pulse (dashed line); both emitters have linewidths of $1.0 \mu \mathrm{eV}$. (d) Average concurrence as a function of normalized emitter detuning $\delta / \Gamma$ for two monochromatic photons (red lines) and a single monochromatic photon (blue lines). Solid lines represent $\beta=1$ and dashed lines represent $\beta=0.9$. The optimal photon frequencies for various coupling and detuning ratios are discussed in the Supplemental Material [27].

with the emitters. Entanglement is then heralded by three detector signatures: two photons in $D_{1}$, two photons in $D_{2}$, or a coincidence count. Using two probe photons leads to a rich structure in the average concurrence, and it is now possible to reach deterministic maximal entanglement for spectrally distinct emitters with finite linewidth. The reason for the two-photon advantage can be determined via inspection of Fig. 3(a), where $\mathcal{C}_{\text {av }}$ is shown as a function of the detuning between the photon energy and the transition energy of the first emitter. In the current example where $\delta=1.0 \mu \mathrm{eV}$, maximum entanglement fidelity occurs for emitters with linewidths of $1.0 \mu \mathrm{eV}$ and input photons with energy $\hbar \omega=E_{1}+\delta / 2$. Comparing this value to Fig. 2(a), this input energy corresponds to the point where the emitter spectra are at half of their maximum intensity. For quasimonchromatic input states, the imparted phase shift is additive in photon number; i.e., for the 


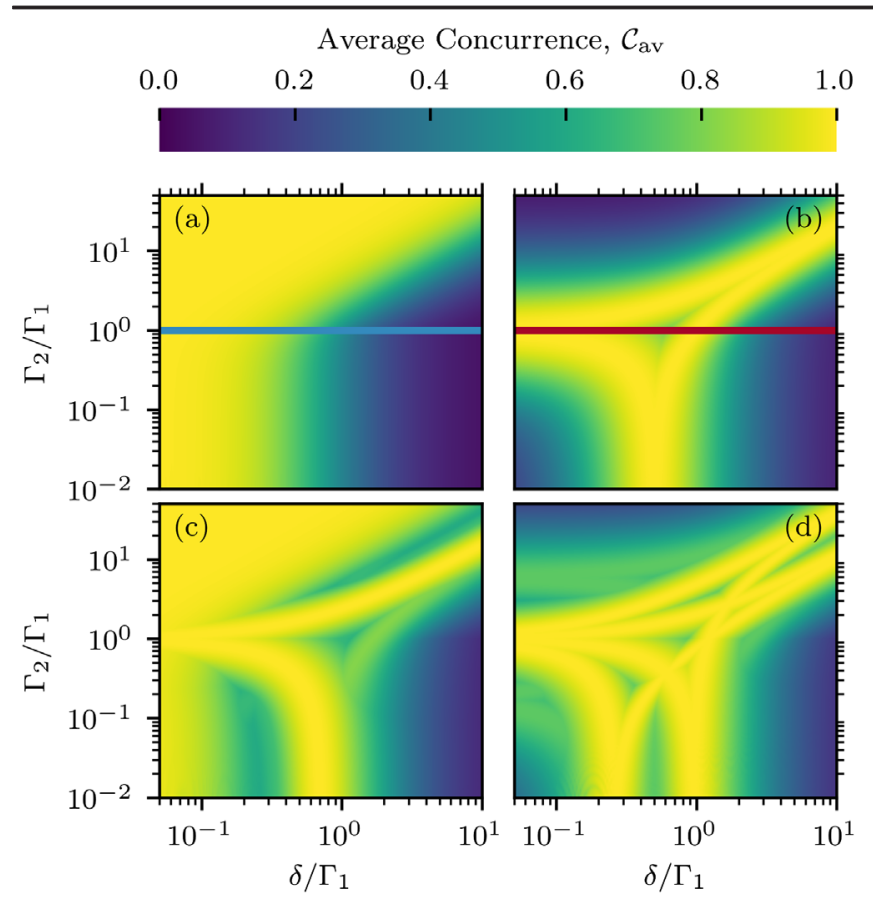

FIG. 4. Maximum average concurrence for different photon number input states injected into the interferometer. The emitter detuning $\delta$ and the linewidth $\Gamma_{2}$ are both normalized to $\Gamma_{1}$, and we consider lossless waveguides $(\beta=1)$. The input photons are identical and monochromatic in the configurations (a) $|1,0\rangle$, (b) $|1,1\rangle$, (c) $|2,1\rangle$, and (d) $|2,2\rangle$. The characteristic shapes in (a) and (b) recur in (c) and (d), and are also found in higher photon number input states $|n, m\rangle$. The blue and red lines in (a) and (b) correspond, respectively, to the solid blue and red lines in Fig. 3(d).

two-photon case, each photon imparts a $\pi / 2$ phase shift to the emitter and therefore achieves the required $\pi$-phase shift. We consider more general emitter detuning and linewidth examples in Fig. 4 and in the Supplemental Material [27].

We study again the effect of broadband probe photons on the entanglement generation process. In this case the phase shift is no longer additive in photon number, since the interaction becomes nonlinear. When two photons are present, the emitter may be excited, which opens a pathway for stimulated emission, such that the coherence of the wave packet is maintained. Competition between spontaneous and stimulated emission processes leads to the nonmonotonic behavior in $\mathcal{C}_{\mathrm{av}}$ presented in Fig. 3(b). The two-photon interaction strength increases when the optical pulse width is broadened to the scale of the emitter linewidth [22]. Although an increased bandwidth generally reduces $\mathcal{C}_{\text {av }}$, a local maximum appears close to the emitter linewidth. We attribute this to the stimulated emission process, which is maximized for photons that are closely matched to the emitters' spectral profile. Figure 3(c) shows the dependence of $\mathcal{C}_{\mathrm{av}}$ on the loss rate $\beta$. In the regime where it is preferable to tune the input photon to the average emitter energy, the effect of reducing the beta factor of either emitter causes the average concurrence to fall in the same manner as if both beta factors were reduced. In the alternative regime, where it is optimal to tune onto one emitter, a difference in beta factors introduces an asymmetry in the behavior of the average concurrence and it becomes preferable to tune onto the emitter that is more efficiently coupled to the waveguide. The two-photon entanglement generation process outperforms the singlephoton process for all values of $\beta$. In Fig. 3(d) we show how two-photon input states can achieve a significant increase in $\mathcal{C}_{\text {av }}$ over single photons for larger detunings.

In certain regimes the two-photon process is capable of generating maximal entanglement between spectrally distinct emitters where the single-photon process fails. We studied the robustness of this effect with respect to the system parameters. In Figs. 4(a) and 4(b) we show the maximum $\mathcal{C}_{\text {av }}$ (optimized over photon frequency $\omega_{\text {opt }}$ ) for the one- and two-photon input states as a function of the emitter detuning and the emitter linewidth ratio. The singlephoton case outperforms the two-photon case if the emitters are spectrally collocated, or if one of the emitters significant overlaps the other, however narrow the linewidth. Crucially, however, by exploiting the multiphoton additivity of the phase shift, a two-photon process can efficiently generate entanglement for any finite detuning without requiring arbitrarily small emitter lifetimes. The converse of this is also true: for any combination of linewidths $\Gamma_{1}$ and $\Gamma_{2}$ there exists a nonzero emitter detuning which creates deterministic maximal entanglement given the optimal two-photon input state. In practice, this means a much greater freedom in matching solid-state emitters for entanglement generation in a Mach-Zehnder interferometer than previously thought.

We extended the entanglement generation process to monochromatic $|n, m\rangle$ Fock states into the interferometer. In Figs. 4(c) and 4(d) we show the maximum $\mathcal{C}_{\mathrm{av}}$ as a function of the emitter detuning and the emitter linewidth ratio for input states $|2,1\rangle$ and $|2,2\rangle$, respectively (for more examples, see the SM [27]). There is a marked improvement in the entanglement generation over the single- and two-photon processes, with larger areas of parameter space achieving a near unity $\mathcal{C}_{\mathrm{av}}$. Remarkably, this indicates that a wide range of imperfections in the fabrication of two identical emitters can be overcome by optical state optimization. Note that the $|2,1\rangle$ case inherits features from both the $|1,1\rangle$ and $|1,0\rangle$ processes. It therefore performs well for both spectrally collocated emitters and those with finite detuning. A similar compound structure is visible in Fig. 4(d), where an input state $|2,2\rangle$ shows a double two-photon structure compared to the $|1,1\rangle$ input in Fig. 4(b). A clear trend emerges, where larger spectral emitter detuning can be overcome by higher number input states $|n, m\rangle$ (see SM [27]).

There are several practical challenges turning this entanglement generation process into a useful quantum 
technology. First, a reduced coupling of the emitter to the waveguide mode will reduce the phase shift imparted on the photons, and therefore lower the average concurrence. Second, the photons may be scattered out of the waveguide mode or be lost in the detection process. However, the use of fast, high-efficiency photon number resolving detectors will mitigate this problem, and such detectors are actively developed [31,32]. Third, the photons must be created in tunable identical quasimonochromatic modes. There are a number of ways this can be achieved over a wide frequency range. Spontaneous parametric down-conversion (SPDC) is inherently tunable [33,34] and frequency filtering will create the optimal quasimonochromatic pulses as well as remove unwanted frequency entanglement. The resulting photon generation rate reduction can be mitigated using multiplexing, which has been demonstrated for both SPDC photon sources [35] and tunable quantum dot sources [36]. Alternatively, tuning of single-photon pulses is possible via frequency conversion [37]. Finally, dephasing will have an impact on the entanglement generation process. The dominant dephasing mechanism for spin-doped solid-state emitters is nuclear spin interactions [38]. While this naturally leads to a random precession of the spin ground state, there are a number of strategies based on dynamical decoupling that may be used to suppress its impact [39-41]. In addition, solid-state emitters are subject to charge fluctuations and phonon interactions. The former leads to spectral wandering occurring on a microsecond timescale, which may be overcome by operating the process on shorter timescales [42]. Phonon scattering leads to sidebands [43] that can be removed through frequency filtering or by placing the emitter in an optical cavity $[44,45]$.

In conclusion, we presented a robust entanglement generation mechanism between two solid-state qubits embedded in a Mach-Zehnder interferometer. Entangling techniques that use solid-state emitters are well known to place very stringent requirements on the spectral identity of the emitters [9]. Our approach overcomes these restrictions by showing how to tailor multiphoton input states, mitigating a long-considered weakness of solid-state emitters. We found that maximal deterministic entanglement between increasingly distinct emitters is possible using higher photon number input states, revealing a rich structure in multiphoton scattering from two emitters with different energies and linewidths. Our work provides a new methodology for solid-state entanglement generation, where the requirement for perfectly matched emitters can be relaxed in favor of optical state optimization.

The authors thank A. P. Foster, D. Hallett, and M. S. Skolnick for useful suggestions, and EPSRC for financial support. K. B. J and J. M. acknowledge funding from the Danish Council for Independent Research (DFF-418100416). J. I.-S. is supported by the Royal Commission for the Exhibition of 1851.
*D. L. H. and K. B. J. contributed equally to this work. p.kok@sheffield.ac.uk

[1] M. A. Nielsen and I. L. Chuang, Quantum Computation and Quantum Information (Cambridge University Press, Cambridge, England, 2000).

[2] G. Wendin, Rep. Prog. Phys. 80, 106001 (2017).

[3] D. Kielpinski, C. Monroe, and D. J. Wineland, Nature (London) 417, 709 (2002).

[4] D. Hallett, A. P. Foster, D. L. Hurst, B. Royall, P. Kok, E. Clarke, I. E. Itskevich, A. M. Fox, M. S. Skolnick, and L. R. Wilson, Optica 5, 644 (2018).

[5] A. Javadi, I. Söllner, M. Arcari, S. L. Hansen, L. Midolo, S. Mahmoodian, G. Kiršanskè, T. Pregnolato, E. H. Lee, J. D. Song, S. Stobbe, and P. Lodahl, Nat. Commun. 6, 8655 (2015).

[6] A. Sipahigil, R. E. Evans, D. D. Sukachev, M. J. Burek, J. Borregaard, M. K. Bhaskar, C. T. Nguyen, J. L. Pacheco, H. A. Atikian, C. Meuwly, R. M. Camacho, F. Jelezko, E. Bielejec, H. Park, M. Lončar, and M. D. Lukin, Science 354, 847 (2016).

[7] P. Lodahl, S. Mahmoodian, and S. Stobbe, Rev. Mod. Phys. 87, 347 (2015)

[8] P. Michler, Single Semiconductor Quantum Dots (Springer, Berlin, 2009).

[9] S. D. Barrett and P. Kok, Phys. Rev. A 71, 060310(R) (2005).

[10] J. Metz and S. D. Barrett, Phys. Rev. A 77, 042323 (2008).

[11] R. Raussendorf and H. J. Briegel, Phys. Rev. Lett. 86, 5188 (2001)

[12] R. Stockill, M. J. Stanley, L. Huthmacher, E. Clarke, M. Hugues, A. J. Miller, C. Matthiesen, C. Le Gall, and M. Atatüre, Phys. Rev. Lett. 119, 010503 (2017).

[13] H. Bernien, B. Hensen, W. Pfaff, G. Koolstra, M. S. Blok, L. Robledo, T. H. Taminiau, M. Markham, D. J. Twitchen, L. Childress, and R. Hanson, Nature (London) 497, 86 (2013).

[14] B. Hensen, H. Bernien, A. E. Dréau, A. Reiserer, N. Kalb, M. S. Blok, J. Ruitenberg, R. F. L. Vermeulen, R. N. Schouten, C. Abellán, W. Amaya, V. Pruneri, M. W. Mitchell, M. Markham, D. J. Twitchen, D. Elkouss, S. Wehner, T. H. Taminiau, and R. Hanson, Nature (London) 526, 682 (2015).

[15] P. Lodahl, S. Mahmoodian, S. Stobbe, A. Rauschenbeutel, P. Schneeweiss, J. Volz, H. Pichler, and P. Zoller, Nature (London) 541, 473 (2017).

[16] I. Söllner, S. Mahmoodian, S. L. Hansen, L. Midolo, A. Javadi, G. Kiršanskè, T. Pregnolato, H. El-Ella, E. H. Lee, J. D. Song, S. Stobbe, and P. Lodahl, Nat. Nanotechnol. 10, 775 (2015).

[17] R. J. Coles, D. M. Price, J. E. Dixon, B. Royall, E. Clarke, P. Kok, M. S. Skolnick, A. M. Fox, and M. N. Makhonin, Nat. Commun. 7, 11183 (2016).

[18] B. Lang, R. Oulton, and D. M. Beggs, J. Opt. 19, 045001 (2017).

[19] D. L. Hurst, D. M. Price, C. Bentham, M. N. Makhonin, B. Royall, E. Clarke, P. Kok, L. R. Wilson, M. S. Skolnick, and A. M. Fox, Nano Lett. 18, 5475 (2018).

[20] S. Fan, S. E. Kocabas, and J.-T. Shen, Phys. Rev. A 82, 063821 (2010). 
[21] A. Roulet and V. Scarani, New J. Phys. 18, 093035 (2016).

[22] A. Nysteen, D. P. S. McCutcheon, M. Heuck, J. Mørk, and D. R. Englund, Phys. Rev. A 95, 062304 (2017).

[23] D. L. Hurst and P. Kok, Phys. Rev. A 97, 043850 (2018).

[24] S. Mahmoodian, P. Lodahl, and A. S. Sørensen, Phys. Rev. Lett. 117, 240501 (2016).

[25] E. Rephaeli and S. Fan, Photonics Res. 1, 110 (2013).

[26] W. K. Wootters, Phys. Rev. Lett. 80, 2245 (1998).

[27] See Supplemental Material at http://link.aps.org/ supplemental/10.1103/PhysRevLett.123.023603 for a description of the "average concurrence" figure of merit, detailed analyses of single, two and N-photon transport through the Mach-Zehnder interferometer and plots showing the optimum optical state energy to employ over a range of the emitter parameter space.

[28] S. Hughes, Opt. Lett. 29, 2659 (2004).

[29] C. K. Hong, Z. Y. Ou, and L. Mandel, Phys. Rev. Lett. 59, 2044 (1987).

[30] A. Nysteen, D. P. S. McCutcheon, and J. Mørk, Phys. Rev. A 91, 063823 (2015).

[31] A. Divochiy, F. Marsili, D. Bitauld, A. Gaggero, R. Leoni, F. Mattioli, A. Korneev, V. Seleznev, N. Kaurova, O. Minaeva, G. Gol'tsman, K. Lagoudakis, M. Benkhaoul, F. Lévy, and A. Fiore, Nat. Photonics 2, 302 (2008).

[32] R. M. Heath, M. G. Tanner, T. D. Drysdale, S. Miki, V. Giannini, S. A. Maier, and R. H. Hadfield, Nano Lett. 15, 819 (2015).

[33] D. N. Klyshko, A. N. Penin, and B. F. Polkovnikov, JETP Lett. 11, 5 (1970).
[34] M. Tanida, R. Okamoto, and S. Takeuchi, Opt. Express 20, 15275 (2012).

[35] C. Joshi, A. Farsi, S. Clemmen, S. Ramelow, and A. L. Gaeta, Nat. Commun. 9, 847 (2018).

[36] H. Wang, Y. He, Y.-H. Li, Z.-E. Su, B. Li, H.-L. Huang, X. Ding, M.-C. Chen, C. Liu, J. Qin, J.-P. Li, Y.-M. He, C. Schneider, M. Kamp, C.-Z. Peng, S. Höfling, C.-Y. Lu, and J.-W. Pan, Nat. Photonics 11, 361 (2017).

[37] Q. Li, M. Davanço, and K. Srinivasan, Nat. Photonics 10, 406 (2016).

[38] I. A. Merkulov, A. L. Efros, and M. Rosen, Phys. Rev. B 65, 205309 (2002).

[39] L. Viola, E. Knill, and S. Lloyd, Phys. Rev. Lett. 82, 2417 (1999).

[40] D. Press, K. De Greve, P. L. McMahon, T. D. Ladd, B. Friess, C. Schneider, M. Kamp, S. Höfling, A. Forchel, and Y. Yamamoto, Nat. Photonics 4, 367 (2010).

[41] A. V. Kuhlmann, J. Houel, A. Ludwig, L. Greuter, D. Reuter, A. D. Wieck, M. Poggio, and R. J. Warburton, Nat. Phys. 9, 570 (2013).

[42] A. Berthelot, I. Favero, G. Cassabois, C. Voisin, C. Delalande, P. Roussignol, R. Ferreira, and J. M. Gérard, Nat. Phys. 2, 759 (2006).

[43] J. Iles-Smith, D. P. S. McCutcheon, J. Mørk, and A. Nazir, Phys. Rev. B 95, 201305(R) (2017).

[44] T. Grange, N. Somaschi, C. Antón, L. De Santis, G. Coppola, V. Giesz, A. Lemaître, I. Sagnes, A. Auffèves, and P. Senellart, Phys. Rev. Lett. 118, 253602 (2017).

[45] J. Iles-Smith, D. P. S. McCutcheon, A. Nazir, and J. Mørk, Nat. Photonics 11, 521 (2017). 